\title{
Using Trityl Carbocations to Introduce Mechanistic Thinking to German High School Students
}

\author{
Catharina Schmitt, Michael Schween* \\ Faculty of Chemistry, Philipps-Universität Marburg, Hans-Meerwein-Straße 4, Marburg, Germany \\ *Corresponding author: schweenm@staff.uni-marburg.de
}

\begin{abstract}
Mechanistic problem-solving is the scientific core competence of organic chemistry. Hence, many students struggle with developing multivariate mechanistic thinking. They very often rely on memorized rules and propose products without providing a detailed mechanistic pathway. They simply apply problem-solving strategies from general chemistry, which is more product-oriented than organic chemistry. A process-oriented view that is highly demanded in organic chemistry requires the understanding and connection of basic principles and concepts. In order to practice the process-oriented approach and introduce advanced German high school students to mechanistic thinking, we developed a set of three new experiments to generate carbocations in model reactions for the observation of reactive intermediates. Trityl cations proved to be the best ones for an experimental investigation of a reaction's progress which is accessible with a simple analysis that generates explicit results by changes in color and electric conductivity. The experiments are arranged in a guided inquiry workshop of six steps alternating theoretical (oral group discussions) and experimental phases.
\end{abstract}

Keywords: high school, First-Year Undergraduate, Laboratory Instruction, organic chemistry, problem-solving, carbocations, conductivity, kinetics, Mechanisms of Reactions, reactive intermediates

Cite This Article: Catharina Schmitt, and Michael Schween, "Using Trityl Carbocations to Introduce Mechanistic Thinking to German High School Students." World Journal of Chemical Education, vol. 6, no. 1 (2018): 18-23. doi: 10.12691/wjce-6-1-4.

\section{Introduction}

Mechanistic problem-solving is one of the most challenging tasks for students in organic chemistry. Current research has shown that only a few advanced learners are actually able to master it, although it is one of the scientific core competences of organic chemistry [1]. Proposing mechanisms is one form of mechanistic problem-solving and it requires deep understanding and connecting of different chemical basic concepts. However, many students fail to predict the mechanism of reactions and simply give the products [2]. This corresponds closely to the thinking in general chemistry, which is mostly product-oriented. Organic chemistry takes a more processoriented view, focusing on the mechanistic pathway between reactants and products [3]. Moving on from general chemistry to organic chemistry, the molecular transformations that are taking place during a reaction move into focus. Students are now asked to apply a less straightforward way to address the products; an addressing of intermediates is required instead. Many students struggle with this transition.

In order to solve problems successfully, multivariate thinking is essential [4]. However, this mechanistic thinking is difficult for students, causing them to often fall back on rote learning [5]. New knowledge is not connected to pre-existing knowledge, which leaves especially novices of organic chemistry with a set of rules that they apply to solve mechanistic problems. They focus on formal familiarities and the determination of products and neglect the mechanistic pathway [6]. This means that the actual mechanism remains a "black box" $[7,8]$. Nevertheless, this use of rule-based reasoning strategies often leads to the correct answer, but a poor conceptual understanding hinders students from transferring their knowledge and solving unknown problems [9-13]. Prior knowledge becomes especially relevant as new information is being closely connected to it [14].

In Germany, basic organic chemistry is already taught in advanced high school courses. These are especially taken by students who want to study chemistry at university in the future. In these courses many basic organic mechanisms like radical reactions, $\mathrm{S}_{\mathrm{N}} 1$ and $\mathrm{E} 1$ mechanisms are taught, but because of the complicated synthesis and the toxicity of many chemicals only a very few experiments are conducted. Additionally, high schools have no access to NMR of GC-MS analyses and hence are unable to determine the formed products of a reaction or even reaction intermediates. This means that mechanisms are often taught in a solely theoretical way which leads to rote memorization and very low mechanistic thinking skills. Nevertheless, mechanistic thinking is one of the most important competences in organic chemistry. As the advanced chemistry courses at high school aim to prepare students for further studies at university, students are at the age of 17-19 when they take these courses, basic training in mechanistic thinking is inevitable. Before the students start with learning organic chemistry, basic 
knowledge in general chemistry has been created for about four years and hence are used to the product-oriented approach of general chemistry.

We developed three key experiments as contrasting cases focusing on carbocations in order to practice the process-oriented view of organic chemistry and to put the focus on the mechanistic pathway and its underlying concepts. The experiments are supposed to first introduce high school students to organic chemistry at university level and aim at practicing basic mechanistic thinking and concluding results and theories from experiments (direct observations). By choosing reactants that form stable intermediates, we created a set of experiments that allow "carbocation watching" [15]. The new experiments make the progress of the reaction visible (the color of the solution changes due to the carbocation formation) and audible (we used an acoustic conductivity device for blind and visually handicapped students). The experiments are simple but meaningful and students can get an easy and experiment-based access to the reaction mechanism. With the spotlight being on the reactive intermediate, students actively engage in making the shift from general chemistry to organic chemistry (Figure 1) [16].
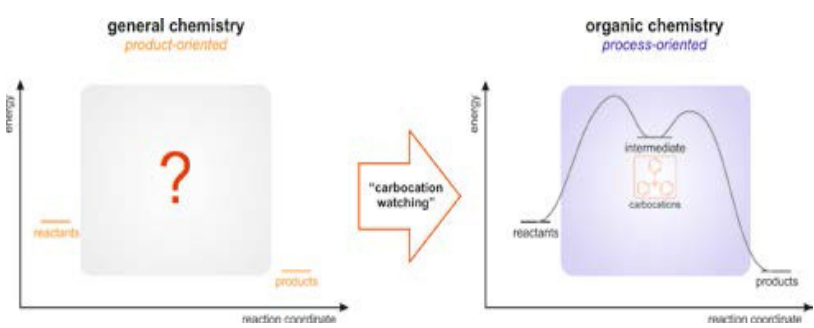

Figure 1. General chemistry takes a more product-oriented view (left), whereas organic chemistry engages in process-oriented thinking (right). The workshop "carbocation watching" focusing on mechanistic pathways is designed to help students make this shift in chemical thinking.

In order to further develop students' mechanistic thinking, we arranged the experiments in a guided inquiry workshop consisting of six steps of alternating theoretical and practical phases to closely assist them in their learning progress. Theoretical interventions (instructor-led in-class discussions) support the stepwise build-up of knowledge on ways of stabilizing electron-pair deficiencies, always in close relationship to the basic chemical concepts of structure-property relationships, donor-acceptor and kinetics. The experiments were conducted by high school students as well as pre-service teachers who might adopt them to their own classes they teach at high school. Though for these purposes the experiments were arranged in a workshop, they can of course be carried out in different contexts, separately or in connection.

\section{Theoretical Background}

Chlorotriphenylmethane ("trityl chloride") and the Lewis acid aluminum(III) chloride are dissolved in dichloromethane and quickly form the stable trityl cation and the tetrachloroaluminate anion (already at $-15{ }^{\circ} \mathrm{C}$ ) (Scheme 1).

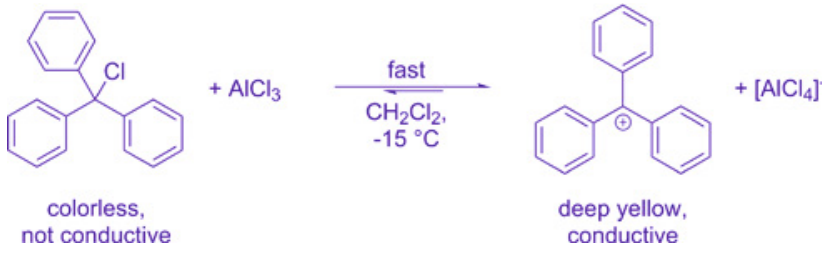

Scheme 1. Reaction of chlorotriphenylmethane with aluminum(III) chloride.

These solutions show easily detectable electric conductivity due to the formation of stable ions. In contrast to the colorless reactant solution, the product solution is an intense deep yellow color, which also allows a close observation of the reaction's progress by UV/Vis spectroscopy. The positive charge is stabilized by resonance. The trityl cation can be represented by ten different resonance forms (Figure 2)

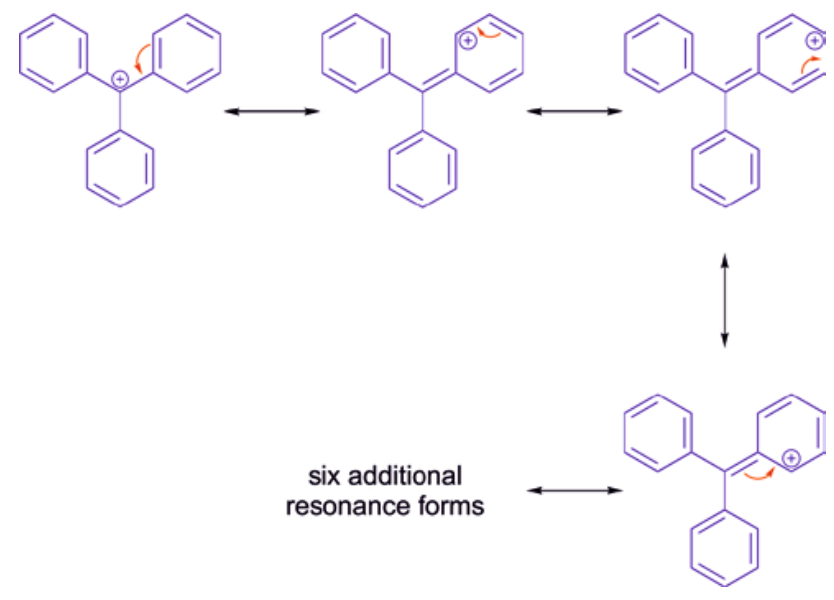

Figure 2. Four out of the ten possible resonance forms for the trityl cation.

Under the same conditions, chlorodiphenylmethane ("benzhydryl chloride") also forms a stable carbocation, but significantly slower (Scheme 2).

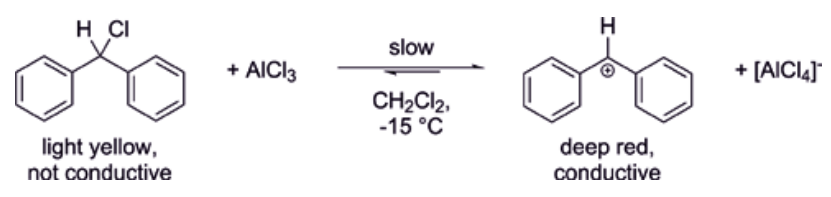

Scheme 2. Reaction of chlorodiphenylmethane with aluminum(III) chloride.

The reason for the different reaction rate is the different stability of the two carbocations generated that form in endergonic reaction steps. The rate-determining step in two-step reactions with cations as intermediates, such as $\mathrm{S}_{\mathrm{N}} 1$ or E1 reactions, is the dissociation of an adequate precursor and the formation of a carbocation. In accordance with Hammond's postulate [17] and the BellEvans-Polanyi principle [18], the energy of the transition states of comparable (endergonic) reactions correlate with the relative energy of the products. This means that the more stable product is formed via a lower transition state and, therefore, faster than a less stable product.

Referring to the two reactions described, it means that the trityl cations form faster than the benzhydryl cations under the same conditions (Figure 3). 


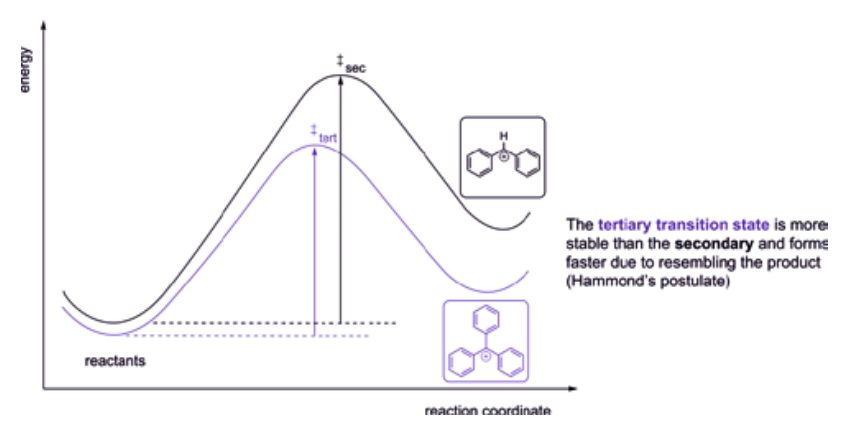

Figure 3. Comparative energy diagram showing the formation of the trityl cation (blue) and the benzhydryl cation (black)

The n-donor substituents (substituents with a "+M-effect") can be inserted, ideally in $4,4^{\prime}$ and 4 " positions, to further increase the stability of the trityl cation's basic framework,. The formation of the $4,4^{\prime}, 4^{\prime \prime}$ trimethoxytriphenylmethyl cation under the conditions given takes place without the addition of aluminum(III) chloride so fast that the rate of formation is not measurable (Scheme 3).
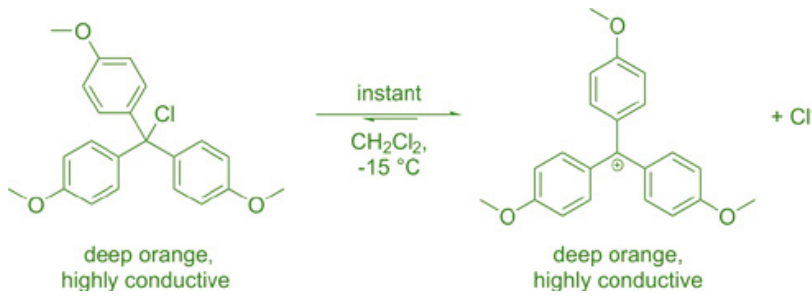

Scheme 3. Reaction of 4,4',4"-trimethoxychlorotriphenylmethane with aluminum(III) chloride

\section{Pedagogic Approach and Goals}

Creating opportunities in which high school students can focus explicitly on reactive intermediates is one approach to help students overcome their struggle in mechanistic problem-solving. The developed experiments deal with one of the most important intermediates in organic chemistry: the carbocation. Carbocations appear in many reactions taught in advanced chemistry courses at high schools and undergraduate courses, for example, $\mathrm{S}_{\mathrm{N}} 1$ reactions, E1 reactions and electrophilic aromatic substitutions [19]. We chose the generation of trityl cations for the workshop because they are extraordinarily stable. This makes them observable with simple analytical methods such as conductivity and the change of color. Measuring changes in conductivity means that carbocations cannot be "produced" by using strong acids (e.g. superacids), but instead we used the Lewis acid aluminum(III) chloride that can be handled easily by students [20]. We have created a reaction that "stops" at the intermediate by the careful selection of reactants. This combines two important goals of the experiments: students experience the importance of considering intermediates for proposing mechanisms and they learn by direct experience because they have to find out about the influence of the underlying principles themselves [21,22].

We arranged the experiments in a six-step workshop and chose a guided inquiry approach to support students use multivariate thinking more confidently [23,24]. In three practical steps students conduct question-driven experiments in small groups [25], the other three steps are oral group discussions led by a lecturer of organic chemistry [26] (Figure 4; a more detailed description of the workshop can be found in the Supporting Information). The alternation of theory and practice allows students to derive hypotheses from experimental data that they discuss firstly in their group and later in class [27]. There is a lot of room for discussions and the students are explicitly allowed to make wrong assumptions and errors [28]. To start with this transfer, the workshop includes three experiments, all following the same structure. In each of them, students have to work together to collect and interpret data, to form hypotheses, and test and apply them to extended contents $[29,30]$.

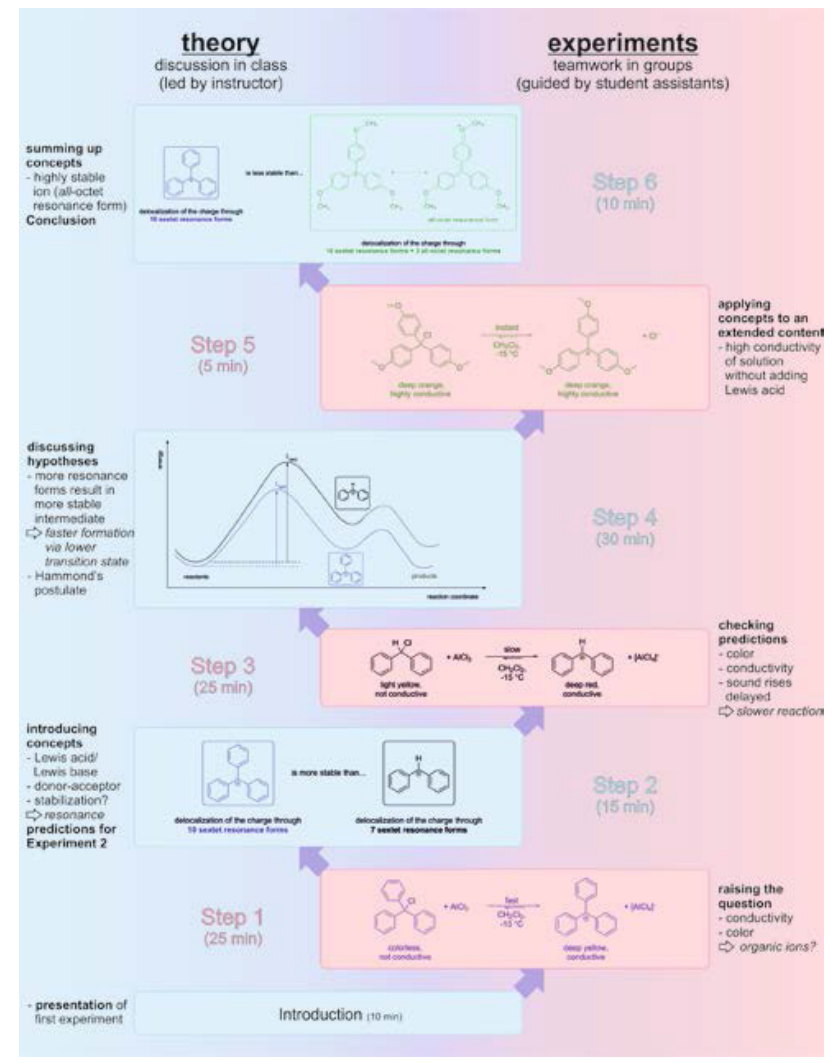

Figure 4. Learning chemical basic concepts by conducting the six-step workshop, interlocking theoretical and practical phases

\section{Experimental Procedure}

\subsection{Experimental Set-up}

Depending on the student's level of understanding, there are many possibilities for measuring conductivity in non-aqueous solutions [31]. The most basic way is the use of an acoustic conductivity measuring device that is used in classes for blind and visually impaired students. The socalled "Chemophon" is commercially available and is very sensitive, especially in solutions with low absolute conductivity [32].

\subsection{Selection of Chemicals}

All the chemicals were selected carefully. We chose chemicals that are nontoxic, easy to handle and inexpensive. Furthermore, all chemicals have to meet 
certain criteria in terms of conductivity. All the chemicals we chose, except potassium hydroxide in ethanol, have a low intrinsic conductivity, so that all increases in conductivity observed are significant and easily detectable. They are checked by the students at the beginning of every experiment to prove the low intrinsic conductivity. We used NMR and UV/Vis spectroscopy to verify our experiments.

\section{Hazards}

All chemicals must be handled with care. All experiments must be conducted under a fume hood with students wearing safety glasses, gloves and a lab coat at all times for safety reasons. Chlorotriphenylmethane and chlorodiphenylmethane can cause severe skin burns and eye damage. Aluminum(III) chloride and 4,4',4"trimethoxychlorotriphenylmethane can cause severe skin burns, eye damage and respiratory irritation. Breathing dust must be avoided. Dichloromethane can cause skin and serious eye irritation and may cause respiratory irritation and drowsiness. Breathing vapors must be avoided. Ethanol is flammable and must be kept away from fire. Potassium hydroxide is harmful if swallowed and can cause severe skin burns and eye damage. Breathing dust or mist must be avoided. Triphenylmethanol, formed as product, can cause skin irritations. Chlorodiphenylmethanol is harmful by inhalation, in contact with skin, or if swallowed and is irritating to the eyes, the respiratory system and to the skin. Therefore, contact with eyes and skin should be avoided.

\section{Results and Discussion}

\subsection{Experiment 1}

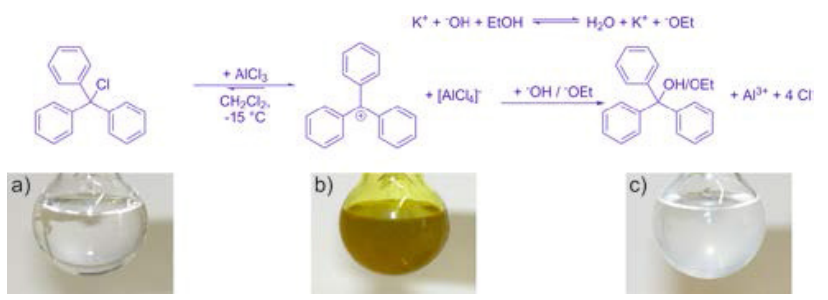

Figure 5. Reaction of chlorotriphenylmethane with aluminum(III) chloride and potassium hydroxide in dichloromethane. a) shows the solution of chlorotriphenylmethane in dichloromethane, b) the solution 2 min after the addition of aluminum(III)chloride and c) the solution after the addition of $3 \mathrm{~mL}$ potassium hydroxide in ethanol

Dichloromethane showed no measurable conductivity. The conductivity of the aluminum(III) chloride solution was low: The Chemophon displayed a buzzing sound. The conductivity of the chlorotriphenylmethane solution was considerably lower: The Chemophon displayed a clicking twice a second. After the addition of chlorotriphenylmethane, the solution turned yellow fast (Figure 5) and the conductivity, and with it the sounds, increased to a loud and high whistling. Conductivity and color did not change until the potassium hydroxide solution was added. With every drop added, the conductivity decreased (the whistling got slower and deeper) and the yellow color slowly vanished. At the end of the "neutralization" of the carbocations with hydroxide/ethoxide ions, conductivity could no longer be detected and the solution was colorless again. The conductivity increased again when more potassium hydroxide solution was added.

\subsection{Experiment 2}

The reaction was equivalent to Experiment 1. The conductivity of the chlorodiphenylmethane solution was very low: The Chemophon displayed a clicking every second. After the addition of chlorodiphenylmethane, the solution turned from light yellow to dark red over the course of $45 \mathrm{~s}$ (Figure 6). The conductivity increased, depicted by the sound rising to a high whistling. When the potassium hydroxide solution was added, the conductivity decreased (the whistling got slower and deeper) and the dark red color slowly vanished with every drop added. At the end of the "neutralization", conductivity could no longer be detected and the solution was light yellow. The conductivity also increased again when more potassium hydroxide solution was added.

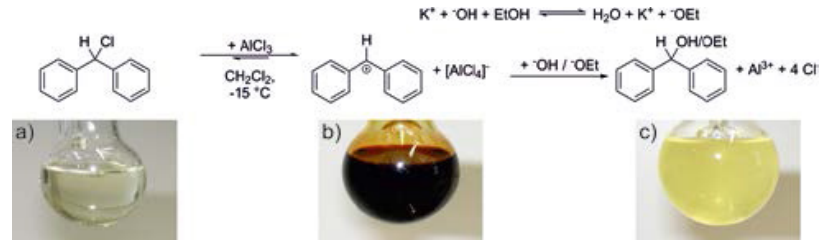

Figure 6. Reaction of chlorodiphenylmethane with aluminum(III) chloride and potassium hydroxide in dichloromethane. a) shows the solution of chlorodiphenylmethane in dichloromethane, b) the solution 2 min after the addition of aluminum(III)chloride and c) the solution after the addition of $3 \mathrm{~mL}$ potassium hydroxide in ethanol.

\subsection{Experiment 3}

The solution of 4,4',4"-trimethoxychlorotriphenylmethane in dichloromethane showed high conductivity at once and the solution was red (Figure 7). The Chemophon displayed a very loud and high whistling and neither sound nor color change. The addition of aluminum(III) chloride did not change that. However, when even a few drops of the potassium hydroxide were added, the conductivity increased at once.
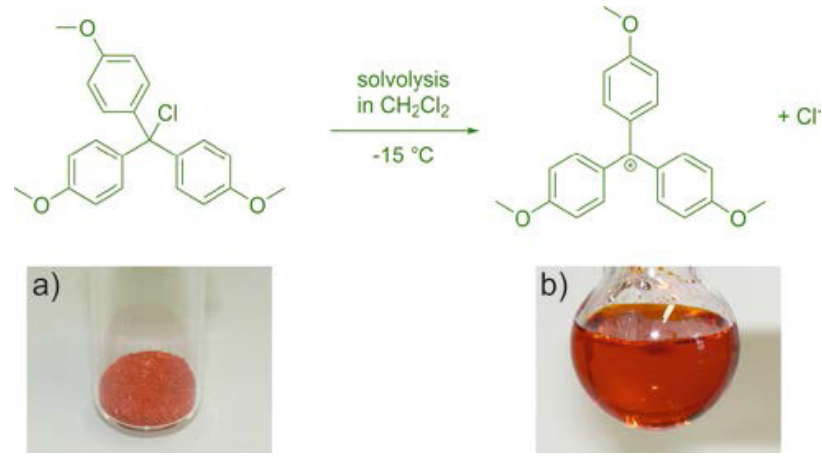

Figure 7. Dissociation of 4,4',4"'-trimethoxychlorotriphenylmethane in dichloromethane. a) shows the solid reactant and b) the reactant dissolved in dichloromethane after $2 \mathrm{~min}$

The experiments were performed 20 times by an overall number of 248 high school students (17-18 years old) and 
31 (pre-service) teachers. The experiments were arranged in the above presented workshop and it took the students two hours to complete it (the time needed for each step can be seen in Figure 4). The students worked in groups of four to five students, where no major difficulties were encountered. All groups achieved the same results that are described above. As none of the students had worked in a laboratory at a university before, high school students were supervised and supported by student assistants. They introduced the students to the Chemophon, explained eventually unknown experimental procedures and answered questions regarding the theoretical parts. Thus, all students were able to perform the experiments independently and were actively engaging in the discussions that followed each experiment. Students got easily used to work with the Chemophon and after using it for the first time they became more secure and conducted the following two experiments faster and with continuously less support.

Conducting the experiments had different goals. Most importantly, introducing advanced high school students to mechanistic thinking. They had all learned various basic organic reactions before (e.g. $\mathrm{S}_{\mathrm{N}} 1$ mechanism, E1 mechanism, electrophilic aromatic substitution, radical reactions), but they had neither taken a closer look at the actual concepts that influence the mechanisms nor performed any experiments that allow them to do so. Experiments 1 and 2 were designed as contrasting cases [33]. Both experiments were performed with only a very short discussion of the results followed by the introduction to the next experiment. Comparison of the results from experiments 1 and 2, students were able to formulate their own hypotheses and verify them in a second experiment. Nevertheless, as they had never performed such advanced experiments and mechanistic thinking, high school students were guided through the experiments by a lecturer and the student assistants. Furthermore, students were supposed to spend a few hours at university in order to conduct more complicated experiments than at school and to get an insight into university.

To assess students' progress in mechanistic thinking, they were asked to answer two prelab and two postlab questions focusing on their way of discussing a proposed mechanism (see Supporting Information). Results showed that in the postlab questions more students were able to answer the questions correctly, but the number of students who used advanced problem-solving strategies taking more concepts into consideration, only increased a little. This is probably due to the fact that high school students encountered this kind of experiments and mechanistic thinking for the first time. The questions as well as the experiments themselves highly rely on the various concepts which underlie the mechanism and which have to be taken into consideration. Although all students participated in the discussions and drew correct conclusions they often still stick to their memorized rules or terms that they acquired during their previous four years of chemical training. A single experiment or workshop can only tackle this problem, but as the results show, it is very beneficial for high school students to start with practicing mechanistic thinking already at high school.

\section{Conclusion and Outlook}

The experiments presented aim at cautiously introducing high school students to mechanistic thinking and fostering their multivariate problem-solving abilities by combining theory and experiment closely in a workshop with a slow and stepwise build-up of knowledge always in connection with previous knowledge. The experiments developed enable students to actively engage in proposing mechanistic pathways and, through this, get access to reaction mechanisms (Figure 8).

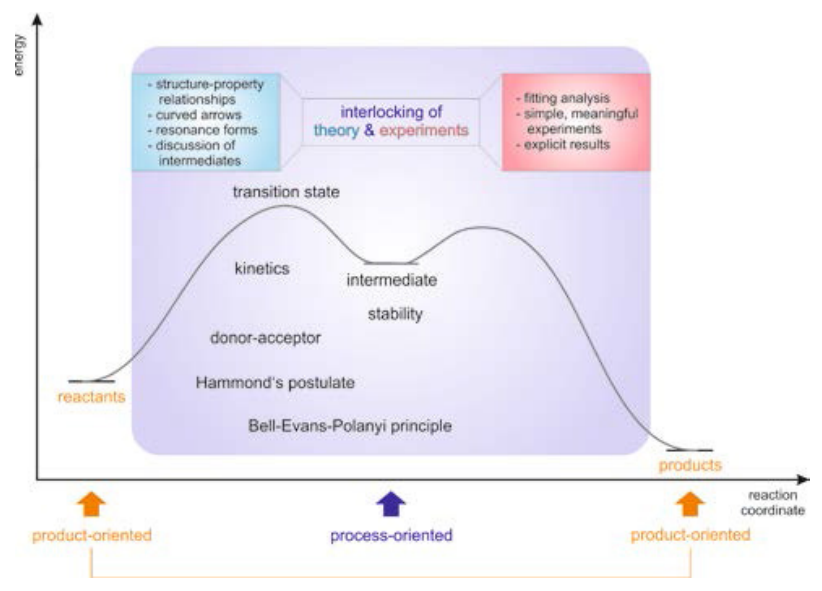

Figure 8. The reaction progress of the formation of trityl cation. Listed are the most important concepts covered in the experiments, focusing on the process-oriented approach

Carbocations are no longer theoretical forms on paper, but compounds that can be measured by conductivity and "seen" due to the changes in color. The theoretical focus is on carbocations and the ways of stabilizing their electronpair deficiency and combines it with the basic concepts of structure-property relationships, relative reaction rate and kinetic considerations.

This knowledge about reaction intermediates and their underlying concepts influencing the mechanistic pathway forms the basis for many other important kinetically controlled organic reactions, such as $\mathrm{S}_{\mathrm{N}} 1$ reactions and electrophilic aromatic substitutions.

Furthermore, students can practice the concept of setting up competitive experiments. These are carried out under the same conditions (in this case Lewis acid, solvent, temperature and concentration), except for one parameter (in this case the reactant). By this procedure, one can find out about the influence of the varied parameters on the reaction's progress. Using this method of two contrasting cases, students get to know a basic method of scientific experimental approach that is also transferable to other mechanistic problems afterwards. The context of carbocation indicators, for example crystal violet, represents a more product-oriented approach, but it can be addressed following the presented experiments.

\section{Acknowledgements}

The authors thank the German Federal Ministry of Education and Research (Grant Number 01PL12037) for supporting this project. 


\section{References}

[1] Graulich, N., "The Tip of the Iceberg in Organic Chemistry Classes: How Do Students Deal with the Invisible?," Chem. Educ. Res. Pract., 16 (1), 9-21. 2015.

[2] Grove, N. P., Copper, M. M., Cox, E. L., "Does Mechanistic Thinking Improve Student Success in Organic Chemistry?," $J$. Chem. Educ., 89 (7), 850-853. 2012.

[3] Ferguson, R., Bodner, G. M., "Making Sense of the ArrowPushing Formalism among Chemistry Majors Enrolled in Organic Chemistry," Chem. Educ. Res. Pract., 9 (2), 102-113. 2008.

[4] Weinrich, M. L., Talanquer, V., "Mapping Student's Modes of Reasoning When Thinking about Chemical Reactions Used to Make a Desired Product," Chem. Educ. Res. Pract., 17 (3), 394-406. 2016

[5] Cooper, M. M., Grove, N., Underwood, S. M., Klymkowsky, M W., "Lost in Lewis Structures: An Investigation of Student Difficulties in Developing Representational Competence," $J$ Chem. Educ., 87 (8), 869-874. 2010.

[6] Flynn, A., "How Do Students Work through Organic Synthesis Learning Activities?," Chem. Educ. Res. Pract., 15 (4), 747-762. 2014.

[7] Anderson, T. L., Bodner, G. M., "What Can We Do about 'Parker'? A Case Study of a Good Student Who Didn't 'Get' Organic Chemistry," Chem. Educ. Res. Pract., 9 (2), 93-101. 2008.

[8] Friesen, J. B., "Saying What You Mean: Teaching Mechanisms in Organic Chemistry," J. Chem. Educ., 85 (11), 1515-1518. 2008.

[9] Kraft, A., Strickland, A. T., Bhattacharyya, G., "Reasonable Reasoning: Multi-Variate Problem-Solving in Organic Chemistry," Chem. Educ. Res. Pract., 11 (4), 281-292. 2010.

[10] Bhattacharyya, G., Bodner, G. M., "Culturing Reality: How Organic Chemistry Graduate Students Develop into Practitioners," J. Res. Sci., 51 (6), 694-713. 2014.

[11] Straumanis, A. R., "Now Bouncing Curved Arrow Technique for the Depiction of Organic Mechanisms," J. Chem. Educ., 86 (12), 1389-1391. 2009.

[12] Grove, N. P., Bretz, S. L., "A Continuum of Learning: From Rote Memorization to Meaningful Learning in Organic Chemistry," Chem. Educ. Res. Pract., 13 (3), 201-208. 2012.

[13] Anzovino, M. E., Bretz, S. L., "Organic Chemistry Students' Ideas about Nucleophiles and Electrophiles: The Role of Charges and Mechanisms." Chem. Educ. Res. Pract., 16 (4), 797-810. 2015.

[14] Vachliotis, T., Salta, K., Vasiliou, P., Tzougraki, C., "Exploring Novel Tools for Assessing High School Students' Meaningful Understanding of Organic Reactions," J. Chem. Educ., 88 (3), 337-345. 2011

[15] Schaller, H. F., Mayr, H., "“Carbocation Watching' in Solvolysis Reactions," Angew. Chem. Int. Ed., 47 (21), 3958-3961. 2008.

[16] Christian, K., Talanquer, V., "Modes of Reasoning in SelfInitiated Study Groups in Chemistry," Chem. Educ. Res. Pract., 13 (3), 286-295. 2012
[17] Hammond, G. S., "A Correlation of Reaction Rates," J. Am. Chem. Soc., 77 (2), 334-338. 1955

[18] Evans, M. G., Polanyi, M., "Further Considerations on the Thermodynamics of Chemical Equilibria and Reaction Rates," Trans. Faraday Soc., 32, 1333-1360. 1936.

[19] Flynn, A. B., Ogilvie, W. W., "Mechanisms before Reactions: A Mechanistic Approach to the Organic Chemistry Curriculum Based on Patterns of Electron Flow," J. Chem. Educ., 92 (5), 803810. 2015.

[20] Olah, G. A., "100 Years of Carbocations and Their Significance in Chemistry." J. Org. Chem., 66 (18), 5943-5957. 2001.

[21] Domin, D. S., "A Review of Laboratory Instruction Styles," $J$. Chem. Educ., 76 (4), 543-547. 1999.

[22] Gaddis, B. A., Schoffstall, A. M., "Incorporating Guided-Inquiry Learning into the Organic Chemistry Laboratory," J. Chem. Educ., 84 (5), 848-851. 2007.

[23] Johnstone, A. H., Al-Shuaili, A., "Learning in the Laboratory; Some Thoughts from the Literature," U. Chem. Ed., (5), 42-51. 2001.

[24] Bhattacharyya, G., "Trials and Tribulations: Student Approaches and Difficulties with Proposing Mechanisms Using the ElectronPushing Formalism," Chem. Educ. Res. Pract., 15 (4), 594-609. 2014.

[25] Mohrig, J. R., "The Problem with Organic Chemistry Labs," J. Chem. Educ., 81 (8), 1083-1085. 2004.

[26] Montes, I., Lai, C., Sanabria, D., "Like Dissolves Like: A Classroom Demonstration and a Guided-Inquiry Experiment for Organic Chemistry," J. Chem. Educ., 80 (4), 447-449. 2003.

[27] Shiland, T. W., "Constructivism: The Implications for Laboratory Work," J. Chem. Educ., 76 (1), 107-109. 1999.

[28] Gallet, C., "Problem-Solving Teaching in the Chemistry Laboratory: Leaving the Cooks...," J. Chem. Educ., 75 (1), $72-77$. 1998.

[29] Farrell, J. J., Moog, R, S., Spencer, J. N., "A Guided Inquiry General Chemistry Course," J. Chem. Educ., 76 (4), 570-574 1999.

[30] Domin, S. D., "A Content Analysis of General Chemistry Laboratory Manuals for Evidence of Higher-Order Cognitive Tasks," J. Chem. Educ., 76 (1), 109-111. 1999.

[31] Newton, T. A., Hill, B. A, Olson, J., "Using Conductivity Devices in Nonaqueous Solutions I: Demonstrating the $\mathrm{S}_{\mathrm{N}} 1$ Mechanism," $J$ Chem. Educ., 81 (1), 58-60. 2004.

[32] Hedinger. https://www.der-hedinger.de/produkte/ph-undleitfaehigkeitsmessung/leitfaehigkeitsmessung/artikel/65361.html. [accessed Oct. 25, 2017].

[33] Schwartz, D., Bransford, A., "A Time for Telling," Cognition and Instruction., 16 (4), 475-522. 1998. 